\title{
Immunobiology
}

ELSEVIER

Immunobiology 211 (2006) 407-417

www.elsevier.de/imbio

\section{CD163 positive subsets of blood dendritic cells: The scavenging macrophage receptors CD163 and CD91 are coexpressed on human dendritic cells and monocytes}

\author{
Maciej Bogdan Maniecki ${ }^{\mathrm{a}}$, Holger Jon Møller ${ }^{\mathrm{a}, *}$, Søren Kragh Moestrup ${ }^{\mathrm{a}, \mathrm{b}}$, \\ Bjarne Kuno Møller \\ ${ }^{a}$ Department of Clinical Biochemistry, Aarhus University Hospital, Norrebrogade 44, DK-8000 Aarhus C, Denmark \\ ${ }^{\mathrm{b}}$ Institute of Medical Biochemistry, University of Aarhus, DK-8000 Aarhus C, Denmark \\ ${ }^{\mathrm{c}}$ Department of Clinical Immunology, Aarhus University Hospital, DK-8000 Aarhus C, Denmark
}

Received 4 May 2006; accepted 23 May 2006

\begin{abstract}
CD163 and CD91 are scavenging receptors with highly increased expression during the differentiation of monocytes into the anti-inflammatory macrophage phenotype. In addition, CD91 is expressed in monocyte-derived dendritic cells (MoDCs), where the receptor is suggested to be important for internalization of CD91-targeted antigens to be presented on the dendritic cell surface for T-cell stimulation. Despite their overlap in functionality, the expression of CD91 and CD163 has never been compared and the expression of CD163 in the monocyte-dendritic cell lineage is not yet characterized. CD163 expression in dendritic cells (DCs) was investigated using multicolor flow cytometry in peripheral blood from 31 healthy donors and 15 HIV-1 patients in addition to umbilical cord blood from 5 newborn infants. Total RNA was isolated from MACS purified DCs and CD163 mRNA was determined with real-time reverse transcriptase polymerase chain reaction. The effect of glucocorticoid and phorbol ester stimulation on monocyte and dendritic cell CD163 and CD91 expression was investigated in cell culture of mononuclear cells using multicolor flow cytometry. We identified two $\mathrm{CD} 163^{+}$subsets in human blood with dendritic cell characteristics, CD $163^{10}$ and $\mathrm{CD} 163^{\text {hi }}$, together constituting a substantial fraction of DCs. Both subsets were characterized as $[\mathrm{lin}]^{-} \mathrm{CD} 4^{+} \mathrm{ILT}^{+}{ }^{+} \mathrm{H}_{-}^{-}$ LA-DR ${ }^{+}$CD $11 \mathrm{c}^{+}$by flow cytometry, and CD163 mRNA was readily detectable in MACS purified human DCs. CD163 on DCs was upregulated by glucocorticoid, and treatment by phorbol ester significantly decreased surface expression. Overall, the expression of CD163 on DCs was significantly increased in HIV-1 patients $(19.3 \%$ [95\% CI: $14.7-26.3 \%])$ compared to healthy patients $(10.5 \%$ [95\% CI: $8.0-12.5]) p<0.001$. The CD $163^{\text {lo }}$ subset was CD $16^{+}$, whereas the $\mathrm{CD} 163^{\text {hi }}$ subset was $\mathrm{CD} 16^{-}$. Both subsets were $\mathrm{CD} 91^{+}$, thereby constituting a subfraction of the recently described $\mathrm{CD} 91^{+} \mathrm{CD} 11 \mathrm{c}^{+}$dendritic cell subset. Coexpression of CD163 and CD91 was also demonstrated on human monocytes, which upon glucocorticoid treatment exhibited an increase in both CD163 and CD91 expression. We have now shown that CD163 and CD91 are coexpressed and coregulated on human monocytes. In addition, two subsets of $\mathrm{CD}_{163}{ }^{+}$DCs constituting a fraction of the recently described $\mathrm{CD} 91^{+} \mathrm{CD} 11 \mathrm{c}^{+}$dendritic cell subset have been
\end{abstract}

\footnotetext{
Abbreviations: $\alpha_{2} \mathrm{M}, \alpha_{2}$ macroglobulin; APC, antigen-presenting cells; DCs, dendritic cells; DEX, dexamethasone; FACS, fluorescence-activated cell sorting; $\mathrm{Hp}-\mathrm{Hb}$, haptoglobin-hemoglobin; Hx-heme, hemopexin-heme; LRP, low density lipoprotein-related protein; mAbs, monoclonal antibodies; MNC, mononuclear cells; PBMC, peripheral blood mononuclear cells; PMA, phorbol 12-myristate 13-acetate

${ }^{*}$ Corresponding author. Tel.: + 4589493450 ; fax: + 4589493060 .

E-mail address: hjmol@as.aaa.dk (H.J. Møller).
} 
identified. The CD163 expression pattern suggests that if antigens are targeted to CD163 they may induce an immunostimulatory response like that of CD91-targeted antigens.

(C) 2006 Elsevier GmbH. All rights reserved.

Keywords: $\alpha_{2}$ macroglobulin; CD163; CD91; Dendritic cells; Monocytes

\section{Introduction}

Dendritic cells (DCs) are bone marrow-derived antigen-presenting cells (APC) that are characterized by their unique capacity to induce and regulate primary immune responses. These rare cells have a remarkable ability to stimulate naïve $\mathrm{T}$ lymphocytes and generate memory $\mathrm{T}$ lymphocytes (MacDonald et al., 2002; Banchereau and Steinman, 1998; Banchereau et al., 2000; Hart, 1997).

Phenotypically, DCs are classified by a high expression of MHC class II molecules and the absence of lineage (Lin)-specific markers (MacDonald et al., 2002; Hart, 1997). Alternatively, DCs can be defined by expression of CD85k/ILT3, high expression of MHC class II and the absence of CD14 (Hart, 1997; MacDonald et al., 2002). At least two distinct human DC subsets, myeloid DCs (DC1) and plasmacytoid DCs (DC2), have been characterized (Wu et al., 2001). DC1 and DC2 are distinct based on their phenotypes $\mathrm{CD} 11 \mathrm{c}^{+} / \mathrm{CD} 123^{\text {lo }}$ and $\mathrm{CD} 11 \mathrm{c}^{-} / \mathrm{CD} 123^{\text {hi }}$, respectively (Robinson et al., 1999).

DC mediated antigen uptake is controlled by a number of dedicated surface molecules, such as Tolllike receptors, scavenger receptors, and mannose receptors that are each responsible for the binding of antigens sharing a common molecular motif through pattern recognition (Banchereau and Steinman, 1998; Hart, 1997; Kaisho and Akira, 2003; Banchereau et al., 2000).

CD91 (LRP, $\alpha_{2}$ macroglobulin $\left(\alpha_{2} \mathrm{M}\right)$ receptor) present on a subset of $\mathrm{CD} 11 \mathrm{c}^{+}$DCs provides a back-up system for internalization of foreign antigens lacking a molecular motif targeted by pattern recognition receptors. CD91 binds activated $\alpha_{2}$ macroglobulin in a complex that includes the foreign activating antigen. Endocytosis of the complex by DCs leads to antigen presentation and activation of T-cells (Hart et al., 2004).

CD91 is also expressed in macrophages and recently, important functions of the two endocytic macrophage receptors CD163 and CD91 have been identified. CD91 is important for clearance of a broad spectrum of ligands including hemopexin-heme (Hx-heme) complexes resulting in cellular heme uptake and lysosomal hemopexin degradation (Hvidberg et al., 2005). In this way, it backs up the yet only known function of CD163, namely to scavenge haptoglobin-hemoglobin $(\mathrm{Hp}-\mathrm{Hb})$ (Kristiansen et al., 2001).

CD163 has been considered to be expressed exclusively on the surface of monocytes and tissue macrophages (Pulford et al., 1992; Backe et al., 1991). The similar functionality of CD91 and CD163 led us to hypothesize that CD163 like CD91 was expressed in DCs. In this work, we present evidence for the existence of two CD163 ${ }^{+}$DC subsets opening the possibility of an immunostimulatory role of CD163 besides its role in hemoglobin scavenging and anti-inflammation.

\section{Materials and methods}

\section{Samples}

EDTA stabilised peripheral blood samples were obtained by standard venipuncture from 15 HIV-1 patients and 31 healthy donors. Furthermore, 5 cord blood samples were collected using umbilical venipuncture. The samples were collected in Venoject ${ }^{\mathbb{R}}$ vacutainers (Terumo Europe NV, Leuven, Belgium). Additionally, leukocyte-rich buffy coats were separated from units of whole blood (approximately $472 \mathrm{ml}$ ) donated by healthy volunteers. The blood was anticoagulated with CPD-A (Baxter, Munich, Germany). The buffy coats were immediately processed as described below.

\section{Monoclonal antibodies}

The following monoclonal antibodies (mAbs) were used in combinations for multicolor flow cytometric analysis ([1] BD Biosciences, San Diego, CA, USA; [2] Diatec.com A/S, Oslo, Norway; [3] DAKO A/S, Glostrup, Denmark; [4] IOTest ${ }^{\circledR}$, Beckman and colter, Marseille, France; [5] Trillium Diagnostics, LLC, Scarborough, Maine, USA; [6] Miltenyi Biotec, Bergisch Gladbach, Germany): Fluoroscein isothiocyanate (FITC)-conjugated anti-CD3 (UCHT1; [1]), antiCD11c (KB90; [3]), anti-CD14 (RMO52; [4]), antiCD16 (3G8; [1]), anti-CD19 (SJ25C1; [1]), anti-CD20 (CAT 13.6E12; [2]), anti-CD56 (NCAM16.2; [1]), antiCD91 (A2MR- $\alpha 2$; [1]) and anti-HLA-DR (EDU-1; [2]); R-Phycoerythrin (R-PE)- conjugated anti-CD163 (GHI/ 61; [1]), anti-CD163 (MAC2-158; [5]) and anti-HLA-DR (EDU-1; [2]); Phycoerythrin-Cy5 (PE-Cy5)-conjugated anti-ILT3/CD85k (ZM3.8; [4]); Peridinin chlorophyll protein (PerCP)-conjugated anti-HLA-DR (L234; [1]); Allophycocyanin (APC)-conjugated anti-CD4 (EDU-2; [2]) and anti-CD14 (18D11; [2]); MicroBeads-conjugated anti-CD14 (TÜK4; [6]), anti-CD19 (LT19; [6]), 
anti-BDCA-3 (AD5-14H12; [6]) and anti-BDCA-4 (AD5-17F6; [6]); Biotin-conjugated anti-CD1c (AD58E7; [6]). R-PE-conjugated Mouse $\mathrm{IgG}_{1}, \kappa$ isotype control (MOPC-21; [1]) was used as a negative control for anti-CD163 PE and analysis for nonspecific antibody binding.

\section{Cell preparation, culture and stimulation}

\section{Human peripheral blood mononuclear cells}

Human peripheral blood mononuclear cells (PBMC) were isolated from blood donor buffy coats by gradient separation using Accuspin System Histopaque ${ }^{\mathbb{R}}$ - 1077 (Sigma-Aldrich Denmark A/S, Broendby, Denmark) according to the manufacturer's protocol.

\section{Dendritic cells}

DCs were purified from PBMC by a 2-step magnetic cell sorting (MACS ${ }^{\circledR}$ technology) using Blood Dendritic Cell Isolation Kit II (Miltenyi Biotec, Bergisch Gladbach, Germany) according to the manufacturer's protocol. In brief, PBMC were incubated with $\mathrm{FcR}$ Blocking Reagent (human IgG) and MicroBeads-conjugated $\mathrm{mAb}$ (anti-CD14 and anti-CD19) and Biotinconjugated anti-CD1c on ice for $15 \mathrm{~min}$, followed by two washes $\left(10 \mathrm{~min}, 4{ }^{\circ} \mathrm{C}, 400 \mathrm{~g}\right)$ in a washing buffer containing PBS (phosphate buffered saline) $\mathrm{pH} 7.2$, $0.5 \%$ bovine serum albumin (BSA) and $2 \mathrm{mM}$ EDTA. Cells were resuspended in washing buffer and passed over an LD Column placed in a magnet in order to deplete non-DCs. The unlabeled pre-enriched DC fraction was then centrifuged $\left(10 \mathrm{~min}, 4^{\circ} \mathrm{C}, 400 \mathrm{~g}\right)$, resuspended in washing buffer and incubated with MicroBeads-conjugated anti-BDCA-3, anti-BDCA-4 and anti-biotin on ice for $15 \mathrm{~min}$, followed by two washes $\left(10 \mathrm{~min}, 4{ }^{\circ} \mathrm{C}, 400 \mathrm{~g}\right)$ in washing buffer. Cells were resuspended in washing buffer and passed over an MS Column placed in a magnet. DCs were recovered by flushing cells out with a plunger after removing the column from the magnet.

\section{Cell culture and stimulation}

PBMC (approximately $1 \times 10^{7}$ cells) were cultured for 3 days in $5 \% \mathrm{CO}_{2}$ and $37^{\circ} \mathrm{C}$ in RPMI 1640 media (RPMI $1640+25 \mathrm{mM}$ HEPES + L-glutamine) (Invitrogen Corporation, Carlsbad, CA, USA) with $20 \%$ FCS. When indicated, $200 \mathrm{nM}$ dexamethasone (DEX) (Merck KGaA, Darmstadt, Germany) was added during culture to increase CD163 expression. In some cultures, cells were finally incubated for $30 \mathrm{~min}$ with $10 \mathrm{nM}$ phorbol 12-myristate 13-acetate (PMA) (Sigma-Aldrich Denmark A/S, Broendby, Denmark), which previously has been shown to induce CD163 shedding. Cells were washed in RPMI 1640 media with $10 \%$ FCS, resus- pended and stained for CD163 expression as described below.

\section{Fluorescence-activated cell sorting (FACS) analysis of cell surface CD163 expression}

Cells $(100 \mu 1$ whole blood or $300 \mu$ PBMC [approximately $3 \times 10^{6}$ cells]) were stained with isotype-matched control antibody or a relevant antibody for $15 \mathrm{~min}$ at room temperature (RT) in the dark. Cells were then washed with $1 \mathrm{ml}$ RPMI 1640 media containing 10\% FCS, resuspended in $400 \mu \mathrm{l}$ FACSflow (Becton Dickinson, San Jose, CA, USA) and analyzed with a FACSCalibur $^{\mathrm{TM}}$ flow cytometer using CELLQuest software $^{\mathrm{TM}}$ (BD Biosciences). At least 100,000 events were acquired to ensure an adequate number of cells for analysis. On a 2-parameter correlated Dot Plot of forward scatter [FSC] versus side scatter [SSC], a gate was set around the mononuclear cells (MNC) clusters. The gated MNC were re-plotted using different 4-color staining protocols and cell definition strategies as indicated in the text. For CD163 density quantitation, flow cytometric estimation of antibodies bound/cell (ABCs) was performed using Quantibrite PE beads (Becton Dickinson, San Jose, CA, USA). The Quantibrite PE beads were run at the same instrument settings as the assay, and the linear regression obtained using the Quantibrite PE beads was used to convert the FL2 linear fluorescence staining of cell population into the number of (CD163) PE molecules bound per cell (ABC). All stainings were controlled using non-specific $\mathrm{mAbs}$, and data was compensated for spectral overlap between fluorochromes using single stained controls and analyzed via FlowJo for Macintosh software Version 6.3 (Tree Star, San Carlos, CA, USA).

\section{Real-time quantitative reverse transcription polymerase chain reaction (RT-PCR) assay}

Total cellular RNA was extracted from whole blood and DCs with QIAamp ${ }^{\circledR}$ RNA Blood Mini (Qiagen, Albertslund, Denmark) according to the manufacturer's protocol, and stored at $-80^{\circ} \mathrm{C}$ until further use.

Reverse transcription was performed by adding $1 \mu$ of the extracted mRNA to a reaction mixture consisting of $2 \mu 110 \times$ PCR buffer II (Applied Biosystems, Naerum, Denmark) supplemented with $6.3 \mathrm{mM} \mathrm{MgCl}_{2}, 0.3 \mathrm{mM}$ of each of the four deoxyribonucleoside triphosphates (dATP, dTTP, dGTP, dCTP), $2.5 \mathrm{mM} 16 \mathrm{mer}$ oligo dT nucleotide, $20 \mathrm{U}$ RNase inhibitor, and $50 \mathrm{U}$ MULV reverse transcriptase in a total volume of $20 \mu \mathrm{l}$ (All reagents from Applied Biosystems, Naerum, Denmark). The cDNA synthesis was carried out in a GeneAmp ${ }^{\mathbb{R}}$ PCR System 9700 Thermal Cycler (Applied Biosystems, Naerum, Denmark) at $42^{\circ} \mathrm{C}$ for $30 \mathrm{~min}$ followed by 
$99^{\circ} \mathrm{C}$ for $5 \mathrm{~min}$. The resulting cDNA provided template for the real-time qPCR assay. The synthesized cDNA was stored at $-20^{\circ} \mathrm{C}$.

Two $\mu 1$ of cDNA were used as template for real-time qPCR in a reaction mixture containing $10 \mathrm{pmol}$ of each primer (CD163 WT; forward primer 5'-ACA TAG ATC ATG CAT CTG TCA TTT G $-3^{\prime}$; reverse primer 5'CAT TCT CCT TGG AAT CTC ACT TCT A-3'; MWG Biotech AG, Edersberg, Germany), $1.0 \mu$ LightCycler $^{\circledR}$ FastStart DNA Master ${ }^{\text {PLUS }}$ SYBR Green I (Roche Diagnostics, Hvidovre, Denmark), containing FastStart Taq DNA Polymerase, reaction buffer, deoxyribonucleoside triphosphates (dATP, dUTP, dGTP, dCTP), SYBR Green I dye, and $10 \mathrm{mM}$ of $\mathrm{MgCl}_{2}$. The volume was adjusted to $10 \mu \mathrm{l}$ with nucleasefree $\mathrm{H}_{2} \mathrm{O}$.

The real-time hot-start qPCR was performed in a LightCycler ${ }^{\circledR}$ System (Roche Diagnostics, Hvidovre, Denmark) with an initial denaturation step of $95^{\circ} \mathrm{C}$ for $15 \mathrm{~min}$, then 50 cycles with a $95^{\circ} \mathrm{C}$ denaturation for $10 \mathrm{~s}$ followed by $65^{\circ} \mathrm{C}$ annealing for $10 \mathrm{~s}$ and $72^{\circ} \mathrm{C}$ extension for $5 \mathrm{~s}$. Amplification specificity was checked by melting curve analysis.

\section{Data and statistical analysis}

Results are given as mean $(95 \%$ CI). Differences between values were analysed for statistical significance with Student's $t$-test. For comparisons between smaller groups without Gaussian distribution of values, the nonparametric Wilcoxon signed-ranks test was used. Differences were considered significant at $p<0.05$. Statistical calculations were performed with SPSS ${ }^{\circledR} 13$ standard version for Windows (SPSS Inc., Chicago, IL) software.

\section{Results}

\section{Identification of CD163 positive peripheral blood cells with dendritic cell characteristics}

Whole blood was stained with specific mAbs defining DCs as $\mathrm{CD}^{-} 4^{-} \mathrm{ILT}^{+}{ }^{+} \mathrm{HLA}^{-} \mathrm{DR}^{+}$and monocytes as $\mathrm{CD}_{14}{ }^{+} \mathrm{ILT}^{+}{ }^{+} \mathrm{HLA}^{-D R}{ }^{+}$. As shown in Fig. 1C, a substantial fraction of monocytes $(88.0 \%$ [95\% CI: 85.0-91.0\%]) stained positive for CD163 (GHI/ $61 \mathrm{mAb}$ ), as expected. The median fluorescence intensity (MFI) was 22.3 (95\% CI: 19.2-25.4). Most DCs stained negative for CD163, however, 10.5\% (95\% CI: 8.0-12.5) stained positive for CD163 (GHI/61 mAb). The $\mathrm{CD} 63^{+}$DCs segregated into two populations; a subset with staining intensity comparable to monocytes $\left(\mathrm{CD} 163^{\mathrm{hi}}, \mathrm{MFI}=34.6[95 \%\right.$ CI: 30.5-40.7]) and a weaker staining subset $\left(\mathrm{CD} 163^{\mathrm{lo}}, \mathrm{MFI}=4.2[95 \% \mathrm{CI}\right.$ : 3.7-5.1]) (Fig. 1C). For comparison, the MFI of an isotype-matched non-specific $\operatorname{IgG}$ staining in the same subset was 1.23 [95\% CI: 1.14-1.46] (Fig. 1D).

In order to exclude unspecific cross-reactivity, the experiments were repeated using an alternative $\mathrm{CD} 163 \mathrm{mAb}$ (MAC2-158). Both the MFI (107.5 [95\% CI: 74.7-140.3] versus 22.3 [95\% CI: 19.2-25.4]) and fraction (32.3 [95\% CI: $19.6-45.1]$ versus 10.5 [95\% CI: 8.0-12.5]) of positive DCs were significantly higher $(p<0.001$ and 0.01 , respectively) using this antibody, however, again two populations of $\mathrm{CD}_{163}{ }^{+} \mathrm{DC}$ were identified (Fig. 1E). Also, the MFI and fraction of positive monocytes were significantly higher using MAC2-158.

DC CD163 gene expression was assessed by reverse transcriptase RT-qPCR using MACS purified DCs from peripheral blood. A significant synthesis of CD163 mRNA was detected in DCs (Fig. 1G).

\section{Surface antigen characteristics of CD163 positive dendritic cells}

To investigate surface antigen characteristics of the two identified $\mathrm{CD}_{163}{ }^{+}$DC populations, CD163, HLADR, ILT3, CD11c, and CD16 expression were investigated by flow cytometric analysis. The $\mathrm{CD} 163^{\mathrm{lo}}$ subset was HLA-DR ${ }^{\text {lo }}$ and ILT $3^{\text {lo }}$, whereas the $\mathrm{CD} 163^{\text {hi }}$ subset was HLA-DR ${ }^{\text {hi }}$ and ILT3 ${ }^{\text {hi }}$ (Figs. 1E and 2A). Both subsets expressed CD11c, which indicates that they both belong to the myeloid subtype (DC1) of DCs (Fig. 2B). Interestingly, only the $\mathrm{CD} 163^{\text {lo }}$ population stained positive for CD16 (the low-affinity receptor for aggregated IgG, Fc $\gamma$ RIII) (Fig. 2C). In order to verify the existence of the two CD163 ${ }^{+}$DC subsets, an alternative gating procedure was used. In this selection, DCs were defined as lineage[CD3,CD14,CD16,CD19,CD20, $\mathrm{CD} 56]^{-} \mathrm{CD} 4{ }^{+}$HLA-DR ${ }^{+}$. Fig. 3D shows the presence of only one $\mathrm{CD} 163^{+}$DC population $\left(\mathrm{CD} 163^{\mathrm{hi}}\right)$. However, when repeating the experiment with the exclusion of the CD16 antibody from the lineage cocktail, both $\mathrm{CD}_{163^{+}}$populations were identified (Fig. 3E, compare with Fig. 1E). Therefore, the $\mathrm{CD} 163^{\mathrm{lo}}$ subset was characterized as being [CD3,CD14,CD19,CD20,CD56] $\mathrm{CD} 4{ }^{+} \mathrm{ILT}^{\mathrm{lo}}{ }^{\mathrm{HLA}} \mathrm{-DR}{ }^{\mathrm{lo}} \mathrm{CD} 11 \mathrm{c}^{+} \mathrm{CD} 16^{+}$and the CD $163^{\mathrm{hi}}$ subset characterized as [CD3,CD14,CD16,CD19,CD20, CD56 ${ }^{-} \mathrm{CD} 4{ }^{+} \mathrm{ILT}^{\mathrm{hi}}{ }^{\mathrm{HLA}} \mathrm{ADR}{ }^{\text {hi }} \mathrm{CD} 11 \mathrm{c}^{+}$. Backgating analysis showed the CD163 ${ }^{+}$DCs $\left(\mathrm{Lin}^{-} \mathrm{CD} 4{ }^{+} \mathrm{HLA}-\right.$ $\mathrm{DR}^{+}$) as a distinct subset of cells localized between lymphocytes and monocytes (Fig. 3G).

\section{Regulation of dendritic cell CD163 expression by glucocorticoid and phorbol ester}

To assess whether different stimuli could influence CD163 expression on DCs as on monocytes, freshly isolated PBMC were cultured for 3 days with or without DEX (3 days) or PMA (30 min). The cells were then 


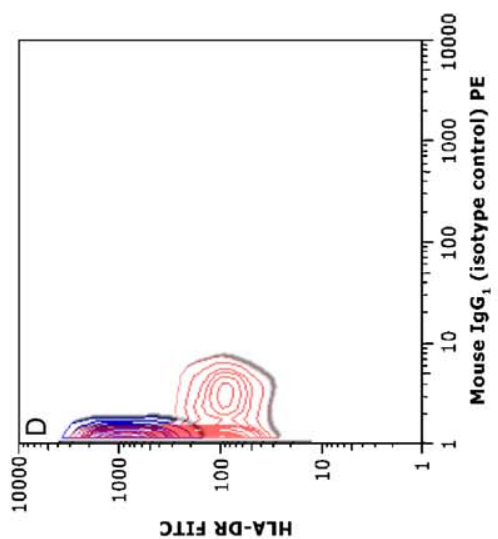

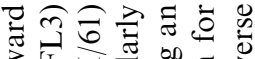

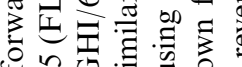

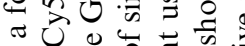

$\exists$ 南

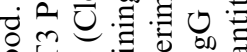

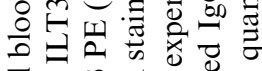

需

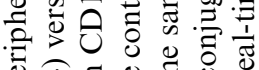

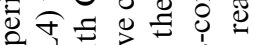

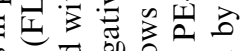

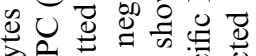

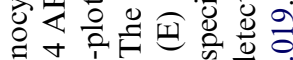

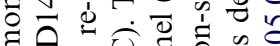

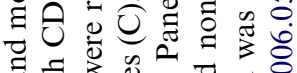

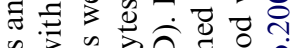

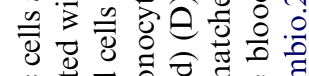

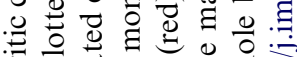

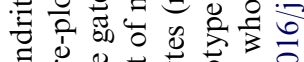

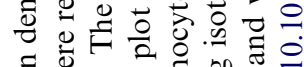

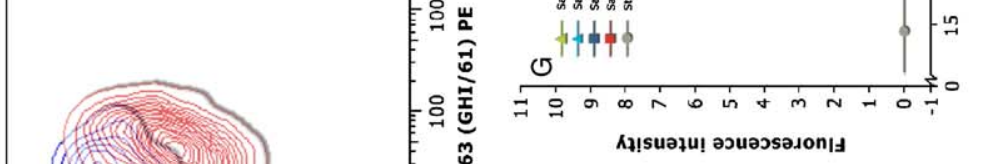

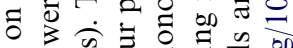

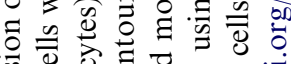
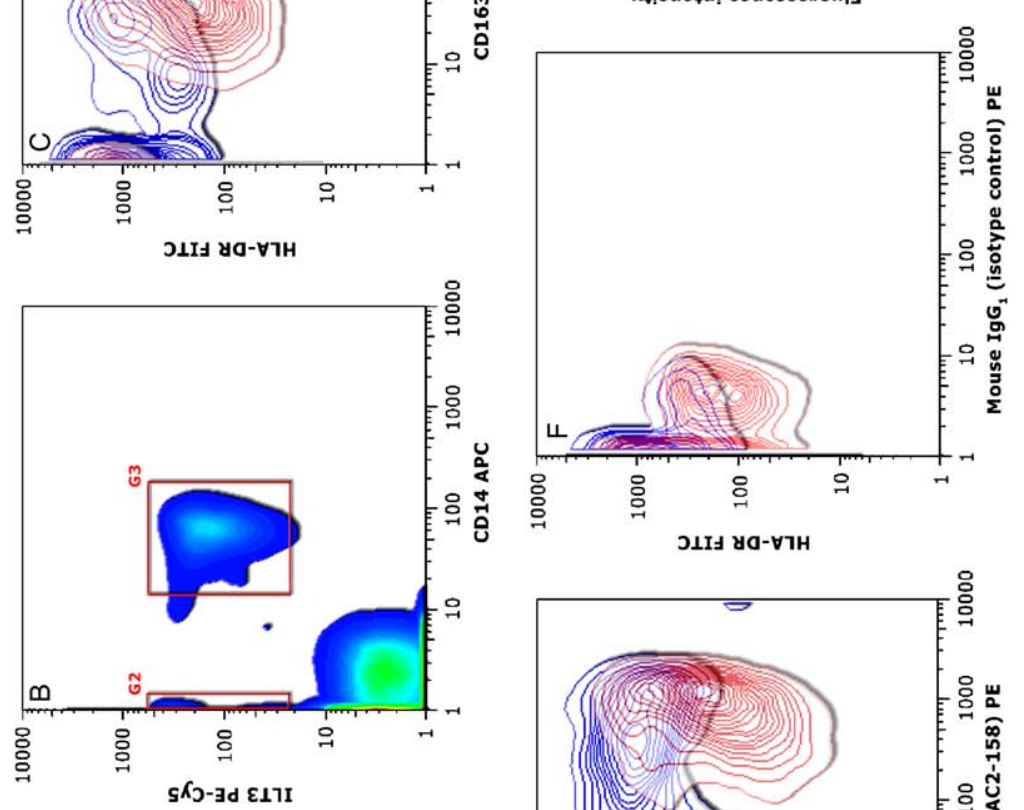

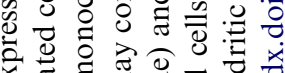

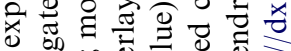

ชิ

$1=0$

U

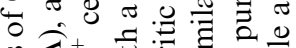

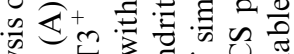

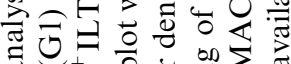

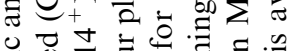

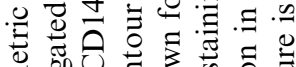

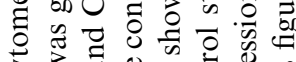

否 $3 \cong$.

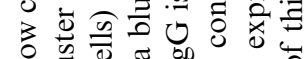

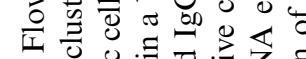

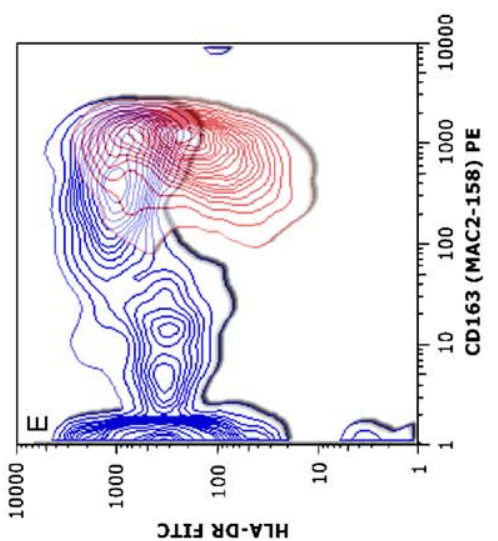

ชె ত

可 0

次

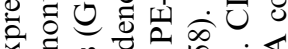

×

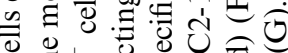

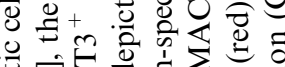

词鸪。

可子可

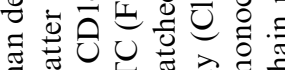

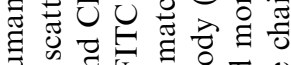

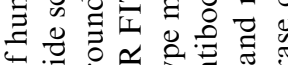

万ั

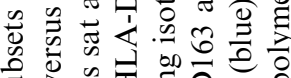

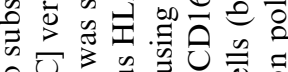

कण

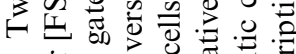

$-\dot{0} \varangle \widehat{T}$

본 

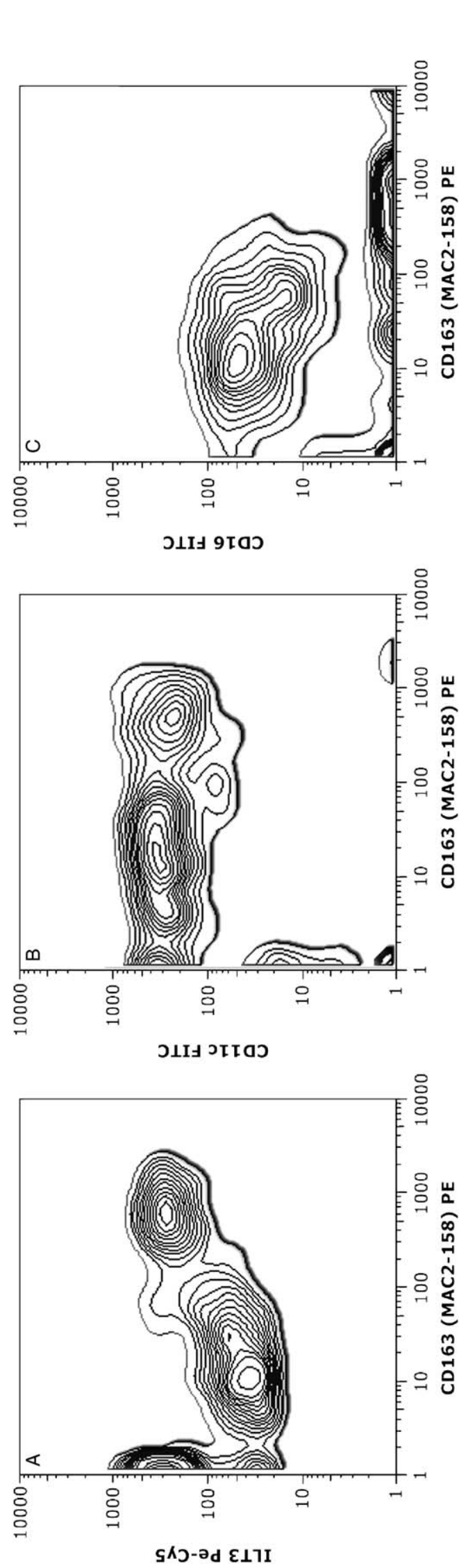

sイว-วd $\varepsilon \perp า I$
$\Xi \widehat{\mathcal{D}} \quad$ stained and subjected to flow cytometry. Analysis of DCs defined as $\mathrm{CD} 14^{-} \mathrm{ILT}^{+}{ }^{+} \mathrm{HLA}-\mathrm{DR}^{+}$showed that CD163 expression increased significantly when stimulated with DEX (Fig. 4). Phorbol ester is known to induce shedding of the extracellular part of CD163 in monocytes, and a significant decrease in CD163 expression was also observed in DCs when treated with PMA (Fig. 4).

\section{Dendritic cell CD163 expression in healthy controls and in HIV-1 infection}

Overall, the fraction of $\mathrm{CD}_{163^{+}}$DCs in HIV-1 infected patients as assessed by the $\mathrm{GHI} / 61$ clone (19.3\% [95\% CI: $14.7-26.3 \%])$ was significantly higher than in healthy controls $(10.5 \%$ [95\% CI: 8.0-12.5]) $(p<0.001)$ (Fig. 5A). Fifteen of 15 HIV-1 infected patients expressed both populations of $\mathrm{CD} 63^{+}$DCs $\left(\mathrm{CD} 163^{\mathrm{lo}}\right.$ and $\left.\mathrm{CD} 163^{\mathrm{hi}}\right)$, whereas 16 of 31 healthy controls only expressed one of the populations (Fig. 5B). Furthermore, in controls that expressed the CD163 ${ }^{\text {hi }}$ subset $(75 \%)$, the mean expression was significantly lower than in the HIV-1 patients. $(p<0.001)$ (Fig. 5C). For comparison, the CD163 expression on monocytes was also determined and showed a significantly higher CD163 expression in HIV-1 infected patients as compared to controls $(p<0.01)$ (not shown).

Additionally, the existence of $\mathrm{CD}_{163}{ }^{+}$DCs was demonstrated in umbilical cord blood from 5 individuals. In one individual, both $\mathrm{CD} 163^{\text {lo }}$ and $\mathrm{CD} 163^{\text {hi }}$ subsets of $\mathrm{CD}_{163}{ }^{+}$DCs were present, whereas only CD163 ${ }^{\text {hi }}$ subset was present in 4 samples (not shown).

\section{Coexpression of CD163 and CD91 on dendritic cells and monocytes}

In the macrophage iron metabolism, key roles for both CD163 and CD91 have been described. As CD91 recently was described on a subset of DCs performing important roles in the adaptive immune responses, we wanted to investigate if the two molecules were coexpressed on monocytes and DCs.

As illustrated in Fig. 6A, flow cytometric analysis showed that DCs $\left(\mathrm{CD} 14^{-} \mathrm{ILT}^{+}{ }^{+} \mathrm{HLA} \mathrm{DR}{ }^{+}\right)$are segregated into at least 4 subsets when whole blood was stained for both CD163 and CD91 (CD91 ${ }^{-} \mathrm{CD} 163^{-}$, $\left.\mathrm{CD} 91^{+} \mathrm{CD} 163^{-}, \mathrm{CD} 91^{+} \mathrm{CD} 163^{\text {lo }}, \mathrm{CD} 91^{+} \mathrm{CD} 163^{\mathrm{hi}}\right)$. This finding indicates that the two $\mathrm{CD} 163^{+}$subsets constitute a subfraction of CD91 ${ }^{+}$DCs. On the other hand, analyzes of monocytes $\left(\mathrm{CD} 14^{+} \mathrm{ILT}^{+}{ }^{+} \mathrm{HLA}-\right.$ $\mathrm{DR}^{+}$), showed one uniform population of $\mathrm{CD} 91^{+}$ $\mathrm{CD} 163^{+}$cells (Fig. 6B).

To investigate whether CD163 and CD91 are coregulated on human monocytes, freshly isolated PBMC were cultured with or without DEX or PMA. 

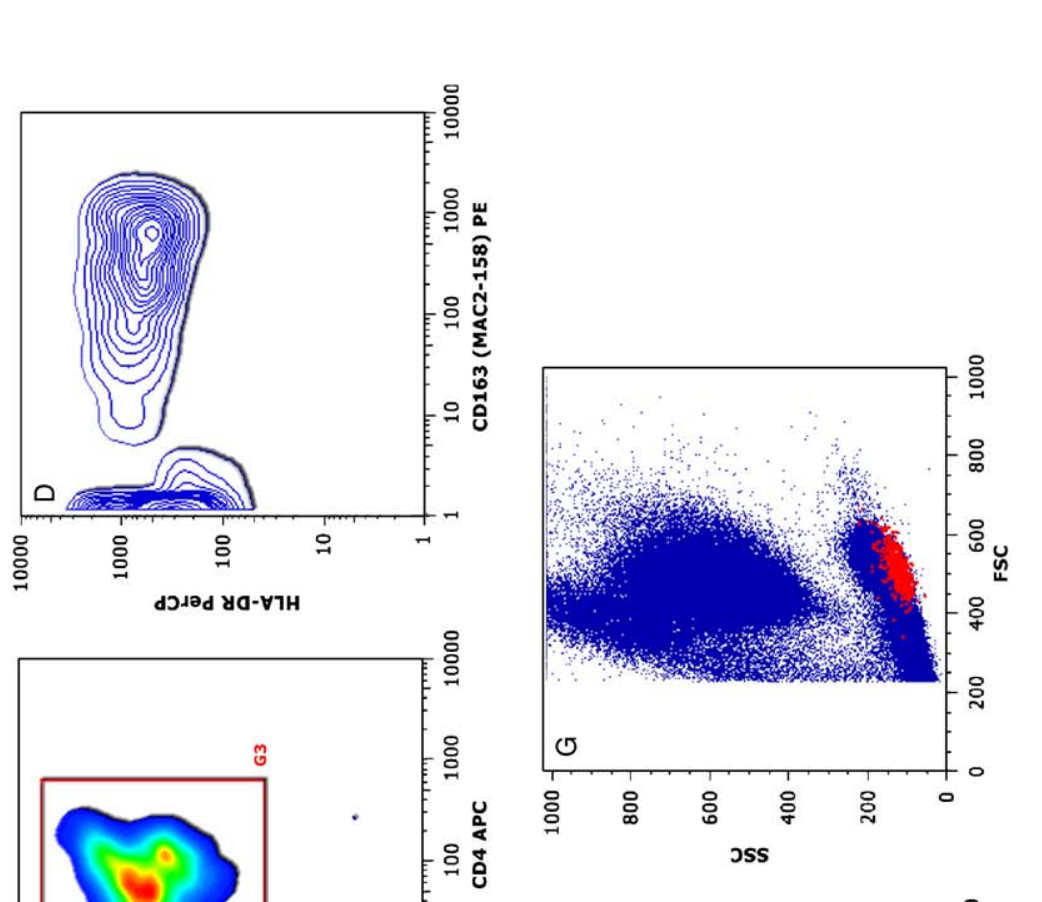

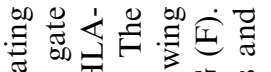

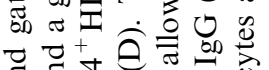

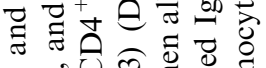

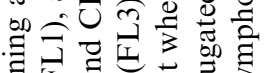

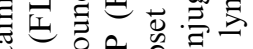

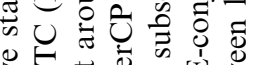

$\sum_{1}$

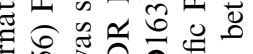

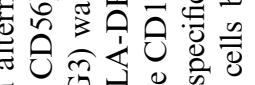

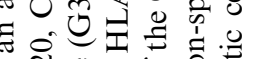

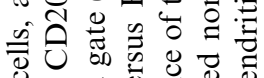

० क क⿺辶े

:

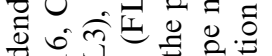

- 正贸 on

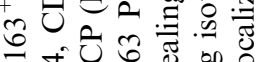

可式용

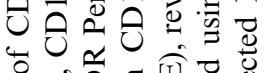

\% คิ

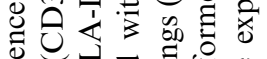

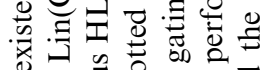

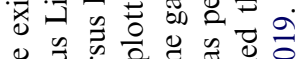

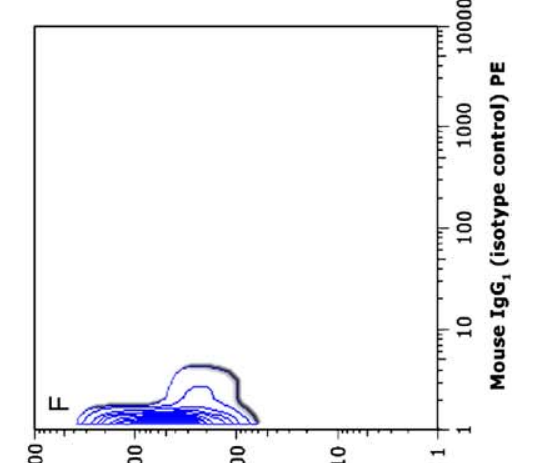

कू

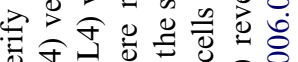

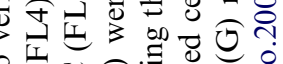

넌을

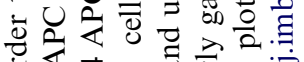

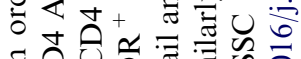

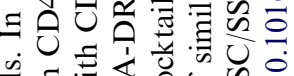

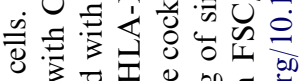

\&

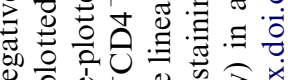

足定

o d.

ष

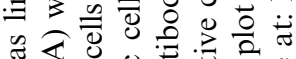
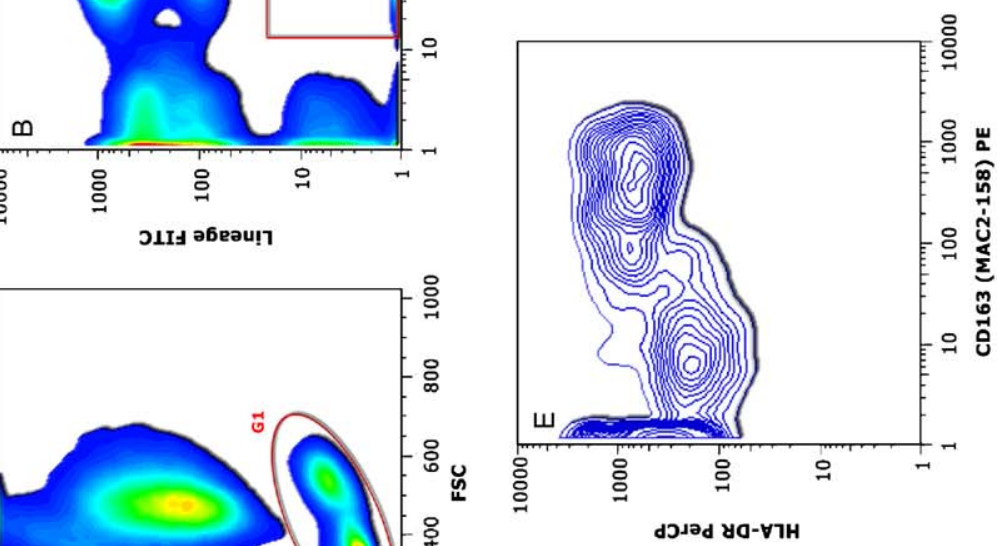

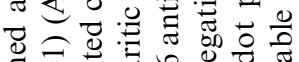

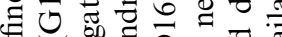

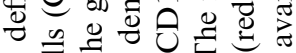

\%

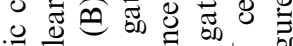

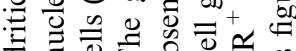

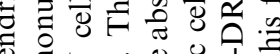

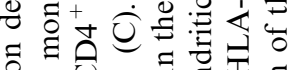

ธี

诺

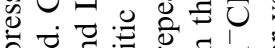

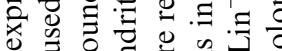

ชิ

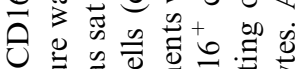

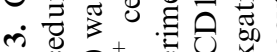

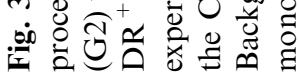




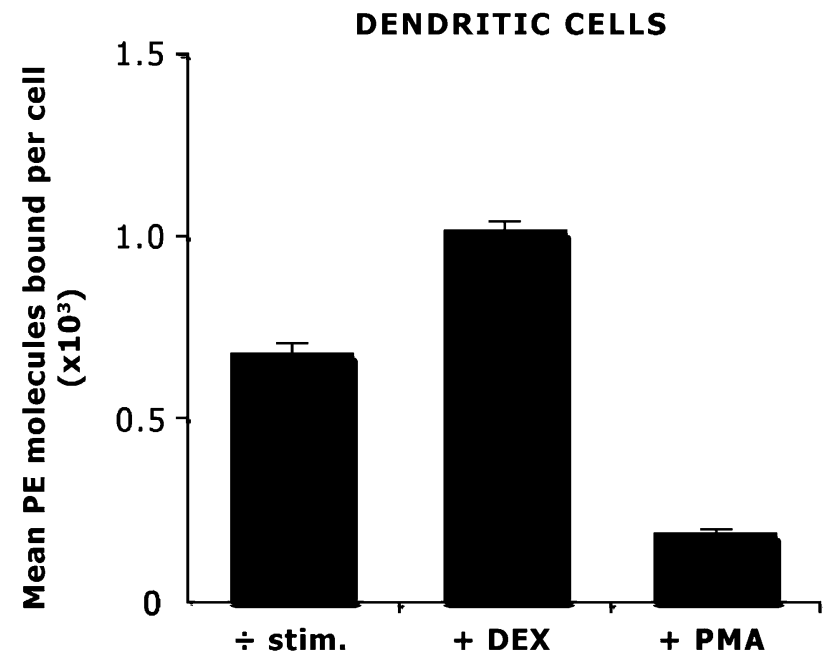

Fig. 4. Effect of glucocorticoid and phorbol ester on dendritic cell CD163 expression. Mononuclear cells were isolated from human whole peripheral blood and cultured for 3 days with or without dexamethasone (DEX) (3 days), or phorbol 12myristate 13-acetate (PMA) (30 min). Washed cells were stained and analysed by flow cytometry. Gated dendritic cells $\left(\mathrm{CD}_{14}{ }^{-} \mathrm{ILT}^{+}{ }^{+}\right)$were re-plotted with CD163 PE (FL2) versus HLA-DR FITC (FL1), and Quantibrite PE beads were used to convert the FL2 linear fluorescence staining of cell population into the number of CD163 PE molecules (GHI/61) bound per cell. Results (representative of three independent experiments) are expressed as mean \pm standard error (SE).

As expected, CD163 expression increased significantly on monocytes $\left(\mathrm{CD} 14^{+} \mathrm{ILT}^{+}{ }^{+} \mathrm{HLA}_{-\mathrm{DR}}{ }^{+}\right)$upon treatment with DEX $(p<0.01)$ and decreased significantly when stimulated with PMA $(p<0.001)$ (Table 1). Interestingly, CD91 expression also increased significantly on monocytes $\left(\mathrm{CD} 14^{+} \mathrm{ILT}^{+}{ }^{+} \mathrm{HLA}^{-\mathrm{DR}^{+}}\right)$when incubated with DEX $(p<0.01)$ and decreased significantly when stimulated with PMA $(p<0.001)$ (Table 1$)$.

\section{Discussion}

We have recently identified $\mathrm{CD} 163$ and $\mathrm{CD} 91$ as essential players in iron-metabolism by scavenging hemoglobin and heme from plasma (Hvidberg et al., 2005; Kristiansen et al., 2001), functions that are linked to secondary anti-inflammatory functions (Moestrup and Moller, 2004). In the present work, we show that the two molecules are coexpressed and coregulated on human monocytes and describe two subsets of $\mathrm{CD}_{163^{+}}$DCs constituting a fraction of the recently described $\mathrm{CD} 91{ }^{+} \mathrm{CD} 11 \mathrm{c}^{+}$DC subset. Monocytes from peripheral blood $\left(\mathrm{CD} 14^{+}\right.$HLA-DR $\left.{ }^{+}\right)$stained positive for both CD163 and CD91 in flow cytometric analysis, and both were significantly upregulated by glucocorti- coid. This coexpression and coregulation seems appropriate in situations of intravascular hemolysis, where the CD163 scavenging system becomes saturated, and free heme in the circulation can be removed by CD91 (Rother et al., 2005).

Besides its role in scavenging heme, CD91 binds and mediates uptake of a multiple other ligands including $\alpha_{2} \mathrm{M}$-proteinase complexes. $\alpha_{2} \mathrm{M}$ becomes activated by foreign proteases which upon cleavage in $\alpha_{2} \mathrm{M}$ become encapsulated, and endocytosed together with $\alpha_{2} \mathrm{M}$ upon binding to CD91 (Moestrup, 1994). CD91 has recently been described on a subset of myeloid-derived DCs, which is of particular interest because endocytosis by the $\alpha_{2}$ M-CD91 pathway in DCs can lead to antigen presentation and T-cell activation (Hart et al., 2004).

CD163 belongs to the class B SRCR receptors that, among others, include CD5, CD6, gp-340, M160, and CD163 (Graversen et al., 2002; Gronlund et al., 2000). Whereas most members are expressed in various cell types and exhibiting a broad range of structurally different ligands, CD163 is expressed selectively in monocytes and tissue macrophages (Pulford et al., 1992; Backe et al., 1991). Currently, there is strong evidence, however, that CD163 also is expressed on leukemic blast cells and monocyte-derived dendritic cells (MoDC) (Walter et al., 2003; Chamorro et al., 2004), and a small subset of $\mathrm{CD} 14^{-} \mathrm{CD} 163^{+}$cells has been identified in peripheral blood that has been interpreted as $\mathrm{CD}^{-}$monocytes (Zarev and Davis, 2004). Although $\mathrm{Hp}-\mathrm{Hb}$ is the only known ligand for CD163 (Kristiansen et al., 2001), the many scavenger receptor repeats in CD163 may function as targets for other ligands. Therefore, it is tempting to hypothesize that CD163 through pattern recognition is involved in processing of foreign antigens.

To further approach this question, we investigated whether CD163, as CD91, is present on subsets of DCs. We identified two subsets of CD163 positive DCs, CD $163^{\text {lo }}$ and $\mathrm{CD} 163^{\text {hi }}$. The two subsets constituted a substantial fraction of peripheral blood DCs in healthy persons. About $10 \%$ of DCs stained positive with the monoclonal anti-CD163 GHI/61, whereas as much as $32 \%$ stained positive with the clone MAC2-158. This difference is probably partly due to sterical hindrance for binding of the GHI/61 antibody, since it binds to domain 7 of CD163, located in proximity to the cell membrane, whereas MAC2-158 binds to domain 1 at the exposed end of CD163 (Madsen et al., 2004). Both subsets were present in approximately $50 \%$ of healthy and in all tested HIV-1 infected patients. Both CD163 ${ }^{+}$ subsets were $\mathrm{CD} 11 \mathrm{c}^{+}$, which refer them to the myeloid lineage (DC1). Furthermore, we demonstrated that the two $\mathrm{CD} 163^{+}$subsets constitute a fraction of the recently described $\mathrm{CD} 1^{+} \mathrm{CD} 11 \mathrm{c}^{+}$DC subset. This emphasizes the relation between CD163 and CD91, since they are coexpressed not only on monocytes but also on DCs. 

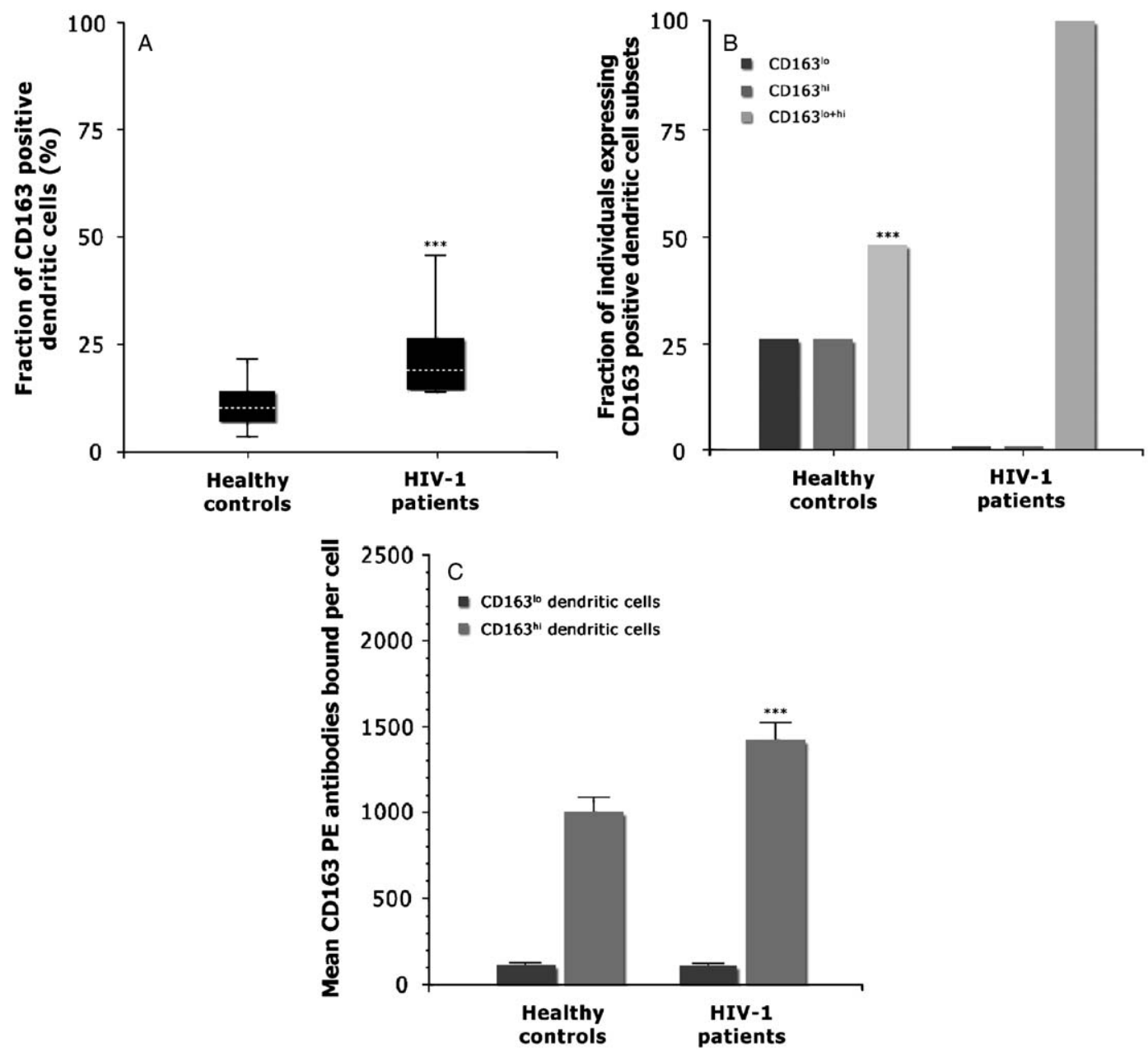

Fig. 5. Dendritic cell CD163 expression in HIV-1 infection. Flow cytometric analysis of CD163 expression on dendritic cells in peripheral blood from HIV-1 patients compared to healthy adults. Gated dendritic cells (CD14- ILT3 ${ }^{+}$) were re-plotted with CD163 PE (FL2) versus HLA-DR FITC (FL1): (A) Fraction of CD163 ${ }^{+}$dendritic cells in normal controls $(n=31)$ and HIV-1 infected patients $(n=15)$. Box plots indicate median, 25-75 percentiles, and range. (B) Fraction of control- and patient-samples expressing the CD163 ${ }^{\text {lo }}$, CD $163^{\text {hi }}$, or both subsets. (C) Level of expression of CD163 in CD163 ${ }^{\text {lo }}$ and CD163 ${ }^{\text {hi }}$ subsets in controls and HIV-1 infected patients. Quantibrite PE beads were used to convert the FL2 linear fluorescence staining of cell population into the number of CD163 PE molecules (GHI/61) bound per cell. Results are expressed as mean \pm standard error (SE). ${ }^{* * *} p<0.001$.

The DC expression of CD163 was upregulated by glucocorticoid as has previously been shown for CD163 expression in monocytes. Moreover, the shedding of CD163 from monocytes by phorbol ester and other inflammatory mediators (Droste et al., 1999) seems to be mirrored in DCs that showed a significant decrease in surface-expression of CD163 after treating with phorbol ester. The identity of the cells as being DCs was confirmed in two different staining protocols; one based on the phenotype CD14 ${ }^{-} \mathrm{ILT}_{3}{ }^{+}$HLA-DR ${ }^{+}$, the other based on Lin $^{-}$HLA-DR ${ }^{+}$.

Heterogeneity of DC phenotypes is well-known, and functional differences between phenotypically distinct subsets are anticipated or in some instances documented. The concept of constitutively inflammatory versus tolerogenic DC subsets has been forwarded, whereas plasticity of the cellular response during induction or resolution of inflammatory processes as recently demonstrated in macrophages is perhaps more likely (Porcheray et al., 2005).

Nevertheless, the correlation of CD163 intensity to ILT3 and HLA-DR surface membrane expression is noteworthy, since ILT3 density in DCs has been shown in vitro to mirror a tolerogenic capacity (Manavalan et al., 2003). If an extrapolation of immunophenotypes obtained in vitro is accepted, weakly CD163 stained DCs in this context might represent an anti-inflammatory subset. The $\mathrm{CD} 16^{+} \mathrm{CD} 163^{\text {lo }}$ subset is ILT3 ${ }^{\mathrm{lo}}$, and $\mathrm{CD}_{16}{ }^{+}$DCs have been reported to constitute a significant proportion of myeloid DCs (MacDonald et al., 2002), and micro array analysis has proposed that toll-like receptor 8 (TLR8) is predominant in these cells (Lindstedt et al., 2005), suggesting a primary role in ssRNA responses. 

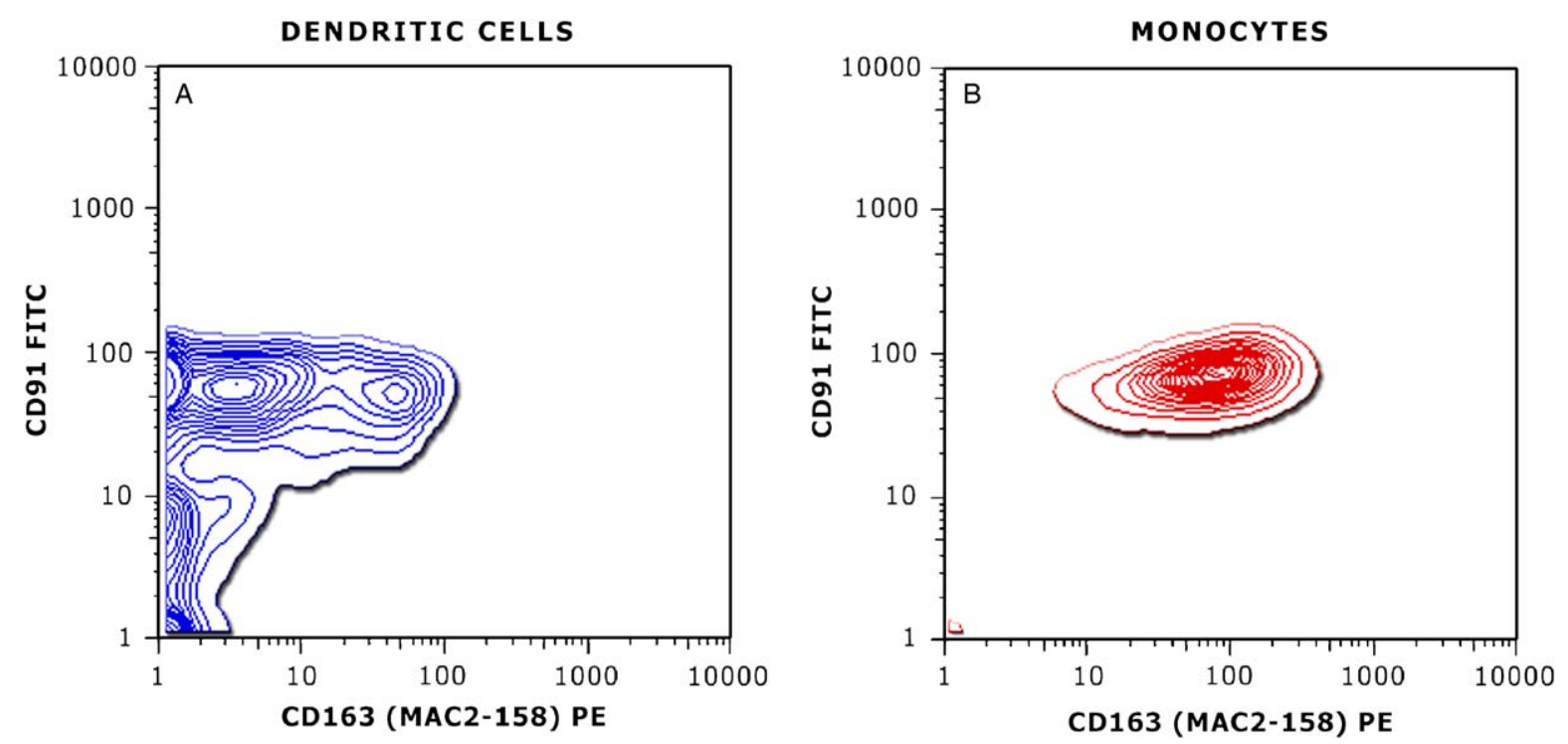

Fig. 6. Coexpression of CD163 and CD91 on monocytes and dendritic cells. Flow cytometric analysis of CD163 and CD91 expression on monocytes and dendritic cells in peripheral blood. Gated dendritic cells (CD14 ${ }^{-}$ILT3 $^{+}$) (A) and monocytes $\left(\mathrm{CD}_{14}{ }^{+} \mathrm{ILT3}^{+}\right)(\mathrm{B})$ were re-plotted with CD163 (MAC2-158) PE (FL2) versus CD91 FITC (FL1). No significant staining was observed using isotype-matched non-specific FITC- and PE-conjugated IgG (not shown).

Table 1. Coregulation of CD163 and CD91 on monocytes

\begin{tabular}{|c|c|c|c|c|c|c|c|c|}
\hline & \multicolumn{8}{|c|}{ Median fluorescence intensity } \\
\hline & Unstimulated & $95 \% \mathrm{CI}$ & + PMA & $95 \% \mathrm{CI}$ & $p$ & $+\mathrm{DEX}$ & $95 \% \mathrm{CI}$ & $p$ \\
\hline CD163 PE & 472.67 & $228.46-716.88$ & 8.80 & $6.53-11.06$ & $<0.01$ & 1211.01 & $1058.21-1363.79$ & $<0.001$ \\
\hline CD91 FITC & 40.07 & $38.49-41.64$ & 27.43 & $23.33-31.53$ & $<0.001$ & 60.33 & $53.57-67.10$ & $<0.001$ \\
\hline
\end{tabular}

Mononuclear cells were isolated from human peripheral blood and cultured for 3 days with or without dexamethasone (DEX) (3 days), or phorbol 12-myristate 13-acetate (PMA) (30 min). Monocytes were stained and analyzed by flow cytometry as described in Fig. 6B. Results are representative of three independent experiments.

Direct targeting of antigens to DCs in vivo via specific surface receptors is a promising method to enhance immunogenicity of vaccines (Gamvrellis et al., 2004). This approach often employs the use of humanized antibodies coupled to the selected antigen (Tacken et al., 2005). The restricted expression of CD163 on DCs and other antigen presenting cells would make CD163 a putative candidate for such strategies. Moreover, CD163 may be targeted not only by antibodies (Van den Heuvel et al., 1999), but also by antigen coupled to $\mathrm{Hp}-\mathrm{Hb}$ complexes or parts thereof capable of binding to the CD163 receptor.

In conclusion, we have shown that CD163 and CD91 are coexpressed and coregulated on human monocytes, and we have identified two subsets of $\mathrm{CD} 163^{+} \mathrm{DC}$ that constitute a fraction of the recently described $\mathrm{CD} 91^{+} \mathrm{CD} 11 \mathrm{c}^{+} \mathrm{DC}$ subset. However, it remains to be shown whether CD163 has a direct immunostimulatory role by binding of foreign antigen as has been demonstrated for the $\alpha_{2}$ M-CD91 pathway.

\section{Acknowledgments}

The authors want to thank Kirsten Bank at the Department of Clinical Biochemistry, Aarhus University Hospital, for excellent technical assistance. Additionally, the authors want to thank Jens Fuglsang, MD, $\mathrm{PhD}$, at the Gynaecological/Obstetrical Research Laboratory, Aarhus University Hospital, for the collected cord blood samples used in this study. This study was partly supported by the Danish Medical Research Council (22-03-0355, HJM).

\section{References}

Backe, E., Schwarting, R., Gerdes, J., Ernst, M., Stein, H., 1991. Ber-MAC3: new monoclonal antibody that defines human monocyte/macrophage differentiation antigen. J. Clin. Pathol. 44, 936-945. 
Banchereau, J., Steinman, R.M., 1998. Dendritic cells and the control of immunity. Nature 392, 245-252.

Banchereau, J., Briere, F., Caux, C., Davoust, J., Lebecque, S., Liu, Y.J., Pulendran, B., Palucka, K., 2000. Immunobiology of dendritic cells. Annu. Rev. Immunol. 18, 767-811.

Chamorro, S., Revilla, C., Gomez, N., Alvarez, B., Alonso, F., Ezquerra, A., Dominguez, J., 2004. In vitro differentiation of porcine blood $\mathrm{CD} 63^{-}$and $\mathrm{CD}^{-} 63^{+}$monocytes into functional dendritic cells. Immunobiology 209, 57-65.

Droste, A., Sorg, C., Hogger, P., 1999. Shedding of CD163, a novel regulatory mechanism for a member of the scavenger receptor cysteine-rich family. Biochem. Biophys. Res. Commun. 256, 110-113.

Gamvrellis, A., Leong, D., Hanley, J.C., Xiang, S.D., Mottram, P., Plebanski, M., 2004. Vaccines that facilitate antigen entry into dendritic cells. Immunol. Cell Biol. 82, 506-516.

Graversen, J.H., Madsen, M., Moestrup, S.K., 2002. CD163: a signal receptor scavenging haptoglobin-hemoglobin complexes from plasma. Int. J. Biochem. Cell Biol. 34, 309-314.

Gronlund, J., Vitved, L., Lausen, M., Skjodt, K., Holmskov, U., 2000. Cloning of a novel scavenger receptor cysteinerich type I transmembrane molecule (M160) expressed by human macrophages. J. Immunol. 165, 6406-6415.

Hart, D.N., 1997. Dendritic cells: unique leukocyte populations which control the primary immune response. Blood 90, 3245-3287.

Hart, J.P., Gunn, M.D., Pizzo, S.V., 2004. A CD91-positive subset of $\mathrm{CD} 11 \mathrm{c}^{+}$blood dendritic cells: characterization of the APC that functions to enhance adaptive immune responses against CD91-targeted antigens. J. Immunol. 172, 70-78.

Hvidberg, V., Maniecki, M.B., Jacobsen, C., Hojrup, P., Moller, H.J., Moestrup, S.K., 2005. Identification of the receptor scavenging hemopexin-heme complexes. Blood 106, 2572-2579.

Kaisho, T., Akira, S., 2003. Regulation of dendritic cell function through Toll-like receptors. Curr. Mol. Med. 3, 373-385.

Kristiansen, M., Graversen, J.H., Jacobsen, C., Sonne, O., Hoffman, H.J., Law, S.K., Moestrup, S.K., 2001. Identification of the haemoglobin scavenger receptor. Nature 409, 198-201.

Lindstedt, M., Lundberg, K., Borrebaeck, C.A., 2005. Gene family clustering identifies functionally associated subsets of human in vivo blood and tonsillar dendritic cells. J. Immunol. 175, 4839-4846.

MacDonald, K.P., Munster, D.J., Clark, G.J., Dzionek, A., Schmitz, J., Hart, D.N., 2002. Characterization of human blood dendritic cell subsets. Blood 100, 4512-4520.

Madsen, M., Moller, H.J., Nielsen, M.J., Jacobsen, C., Graversen, J.H., van den Berg, T., Moestrup, S.K., 2004. Molecular characterization of the haptoglobin.hemoglobin receptor CD163. Ligand binding properties of the scaven- ger receptor cysteine-rich domain region. J. Biol. Chem. 279, 51561-51567.

Manavalan, J.S., Rossi, P.C., Vlad, G., Piazza, F., Yarilina, A., Cortesini, R., Mancini, D., Suciu-Foca, N., 2003. High expression of ILT3 and ILT4 is a general feature of tolerogenic dendritic cells. Transpl. Immunol. 11, 245-258.

Moestrup, S.K., 1994. The alpha 2-macroglobulin receptor and epithelial glycoprotein-330: two giant receptors mediating endocytosis of multiple ligands. Biochim. Biophys. Acta 1197, 197-213.

Moestrup, S.K., Moller, H.J., 2004. CD163: a regulated hemoglobin scavenger receptor with a role in the antiinflammatory response. Ann. Med. 36, 347-354.

Porcheray, F., Viaud, S., Rimaniol, A.C., Leone, C., Samah, B., Dereuddre-Bosquet, N., Dormont, D., Gras, G., 2005. Macrophage activation switching: an asset for the resolution of inflammation. Clin. Exp. Immunol. 142, 481-489.

Pulford, K., Micklem, K., McCarthy, S., Cordell, J., Jones, M., Mason, D.Y., 1992. A monocyte/macrophage antigen recognized by the four antibodies GHI/61, Ber-MAC3, KiM8 and SM4. Immunology 75, 588-595.

Robinson, S.P., Patterson, S., English, N., Davies, D., Knight, S.C., Reid, C.D., 1999. Human peripheral blood contains two distinct lineages of dendritic cells. Eur. J. Immunol. 29, 2769-2778.

Rother, R.P., Bell, L., Hillmen, P., Gladwin, M.T., 2005. The clinical sequelae of intravascular hemolysis and extracellular plasma hemoglobin: a novel mechanism of human disease. J. Am. Med. Assoc. 293, 1653-1662.

Tacken, P.J., de Vries, I., Gijzen, K., Joosten, B., Wu, D., Rother, R.P., Faas, S.J., Punt, C.J., Torensma, R., Adema, G.J., Figdor, C.G., 2005. Effective induction of naive and recall $\mathrm{T}$-cell responses by targeting antigen to human dendritic cells via a humanized anti-DC-SIGN antibody. Blood 106, 1278-1285.

Van den Heuvel, M.M., Tensen, C.P., van As, J.H., Van den Berg, T.K., Fluitsma, D.M., Dijkstra, C.D., Dopp, E.A., Droste, A., Van Gaalen, F.A., Sorg, C., Hogger, P., Beelen, R.H., 1999. Regulation of CD 163 on human macrophages: cross-linking of CD163 induces signaling and activation. J. Leukoc. Biol. 66, 858-866.

Walter, R.B., Bachli, E.B., Schaer, D.J., Ruegg, R., Schoedon, G., 2003. Expression of the hemoglobin scavenger receptor (CD163/HbSR) as immunophenotypic marker of monocytic lineage in acute myeloid leukemia. Blood 101, 3755-3756.

Wu, L., Vandenabeele, S., Georgopoulos, K., 2001. Derivation of dendritic cells from myeloid and lymphoid precursors. Int. Rev. Immunol. 20, 117-135.

Zarev, P.V., Davis, B.H., 2004. Comparative study of monocyte enumeration by flow cytometry: improved detection by combining monocyte-related antibodies with anti-CD163. Lab Hematol. 10, 24-31. 